\title{
Magnetic investigation of low temperature phase transition in iron selenides
}

\author{
Baluyan Tigran ${ }^{1, *}$, Novakova Alla ${ }^{1}$, Khairullin Marat ${ }^{1}$ \\ ${ }^{1}$ Moscow M.V. Lomonosov State University, Department of physics, Russia
}

\begin{abstract}
Fe}_{7} \mathrm{Se}_{8}$ contain large amount of ordered vacancies that define its magnetic structure. Combined comparative magnetic and Mossbauer investigation were performed to find out the peculiarities of the temperature magnetic transitions in these compounds. It was found that adding an additional layer with ordered vacancies in $4 \mathrm{c}$ superstructure increased the temperature of spin rotation.
\end{abstract}

\section{Introduction}

Vacancies can strongly change material properties, which is important for producing lithium-ion batteries, solar cell units, photocatalytical agents etc[1,2]. As it was previously found out in our work [3], $\mathrm{Fe}_{7} \mathrm{Se}_{8}$ with $3 \mathrm{c}$ superstructure shows a special behaviour in electrical resistivity in a temperature range from 30 to $100 \mathrm{~K}$, which leads to a metal-insulator phase transition. It occurs because vacancies are ordered in the selenide structure. It was also shown that this phase transition is preceded by a magnetic phase transition that occurs at $120 \mathrm{~K}$. This magnetic transition is accompanied by the spin rotation. $3 \mathrm{c}-\mathrm{Fe} 7 \mathrm{Se} 8$ is ferrimagnetic above $120 \mathrm{~K}$ $[4,5]$, but lowering the temperature causes spin rotation towards $\mathrm{C}$ axis. As vacancies are strongly ordered in this structure and define its magnetic properties, it was interesting to make a comparison of the observed effect in the same structure with additional atomic layers with vacancies - 4c-Fe7Se8 (Fig.1).
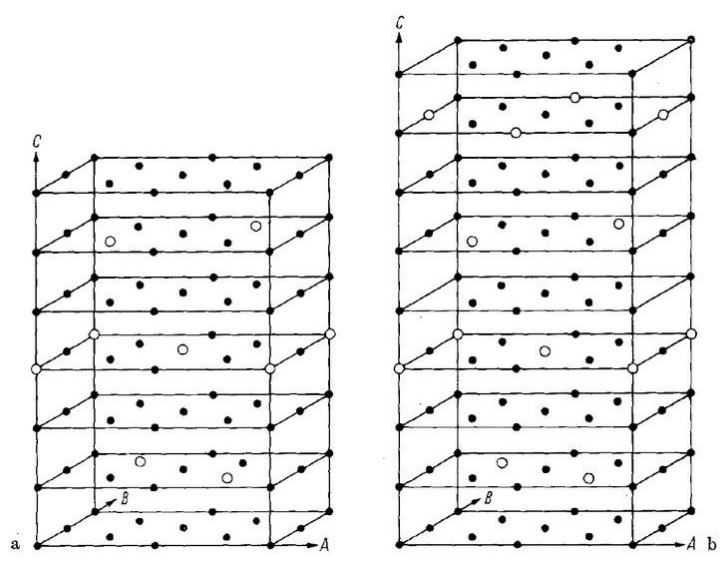

Fig.1. Structure of Fe7Se8 with 3c (left) and 4c (right) superstructure. Black dots are iron atoms, white dots are vacancies. Selenium atoms are omitted for clarity.

Vacancies added in a 4c structure are placed between the layers with no vacancies and next to the diagonal vacancies layer [6]. It was interesting to define the magnetic properties of $4 \mathrm{c}$ structure and apply
Mossbauer and magnetic methods to define the magnetic peculiarities of this compound

\section{Experimental}

High purity $3 \mathrm{c}-$ and $4 \mathrm{c}-\mathrm{Fe}_{7} \mathrm{Se}_{8}$ samples were investigated in this work. Pure powders (99.999\% purity) of Iron and Selenium were sealed in the quartz tube at heated to $1200^{\circ} \mathrm{C}$ and kept at this temperature for an hour. Then the temperature was lowered to $800^{\circ} \mathrm{C}$ and held for another hour. Then it was slowly quenched in order to obtain $3 \mathrm{c}$ superstructure or quickly cooled to room temperature to obtain $4 \mathrm{c}$ superstructure.

Comparative magnetic measurement and Mossbauer investigation was performed for $3 \mathrm{c}$ and $4 \mathrm{c}$ structures to find out the difference of magnetic phase transitions.

Magnetic investigation was performed on the VSM LakeShore 7407 magnetometer in a temperature range from $80 \mathrm{~K}$ to $320 \mathrm{~K}$. The external field applied was $1 \mathrm{kOe}$. We also obtained the hysteresis loops for both structures at a temperature range from $80 \mathrm{~K}$ to $300 \mathrm{~K}$ to find out the temperature of magnet phase transition completion.

Mossbauer investigation was performed on a MS1104Em Mossbauer spectrometer in a temperature range from $80 \mathrm{~K}$ to $300 \mathrm{~K}$. The obtained spectra were analysed using the UnivemMS tool, which allows spectra fitting with lorentzians and calculates Mossbauer parameters e.g. effective magnetic fields.

\section{Results and discussion}

\subsection{Magnetic measurements}

Magnetic moment measurements for $3 \mathrm{c}-$ and $4 \mathrm{c}-\mathrm{Fe}_{7} \mathrm{Se}_{8}$ are presented on figures 2 and 3 respectively. Both of them show the similar behaviour - abrupt drop at a certain temperature range : $100-120 \mathrm{~K}$ for $3 \mathrm{c}$ and 100 $135 \mathrm{~K}$ for $4 \mathrm{c}$. The magnitude of magnetic moment is five time higher for $4 \mathrm{c}$ structure $(0.25$ compared to 0.038 emu). Magnetic moment of $4 \mathrm{c}$ structure

\footnotetext{
Corresponding author tarinax@yandex.ru
} 


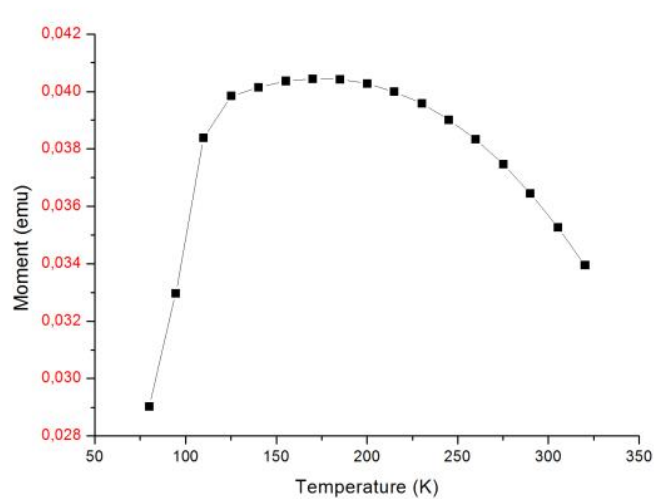

Fig.2. Magnetic moment measurements for $3 \mathrm{c}-\mathrm{Fe}_{7} \mathrm{Se}_{8}$

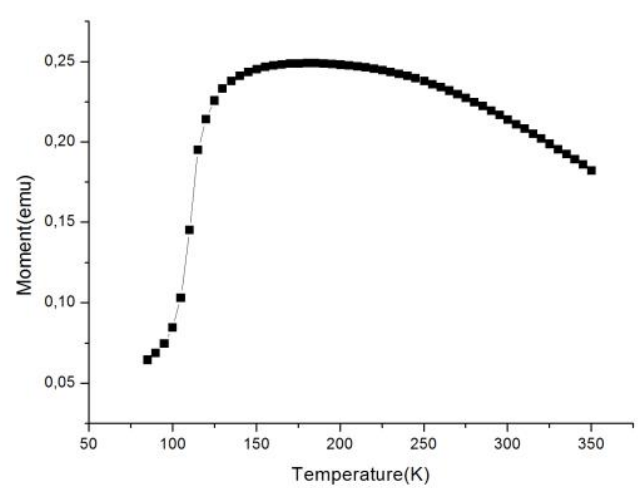

Fig.3. Magnetic moment measurements for $4 c-\mathrm{Fe}_{7} \mathrm{Se}_{8}$

is greater than $3 \mathrm{c}$ because additional atomic layers increase the spin influence of the corresponding ferrimagnetic sublattices[7]. The temperature drop indicates the magnetic phase transition beginning, which is caused by spin rotation toward $\mathrm{C}$ axis in both superstructures. This phase transition leads to anisotropy increasing [8], which allows system to reduce it's electric resistivity with further temperature lowering.

This spin rotation, however is not instant, it begins at the drop temperature ( $120 \mathrm{~K}$ for $3 \mathrm{c}$ and $135 \mathrm{~K}$ for $4 \mathrm{c}$ ) and finishes at temperatures around $85 \mathrm{~K}$. It is known because all the hysteresis loops for both $3 \mathrm{c}$ and $4 \mathrm{c}$ superstructures remained classically shaped for all temperatures until $85 \mathrm{~K}$. Corresponding hysteresis loops are strongly distorted. This distortion shows the completion of magnetic phase transition in the whole structure[9].

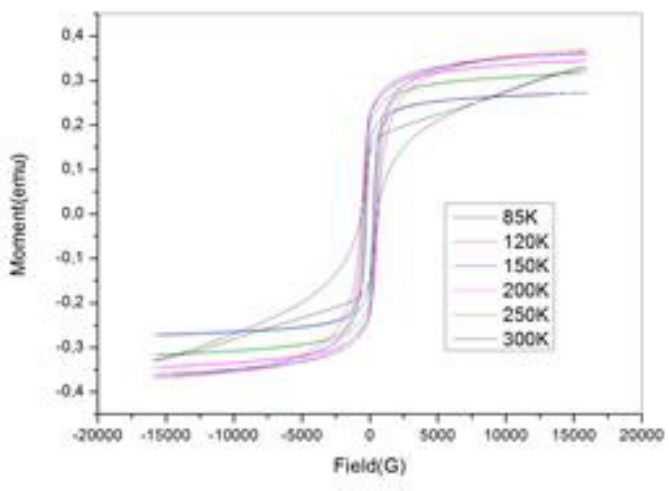

Fig.4. Hysteresis loops for $4 \mathrm{c}-\mathrm{Fe}_{7} \mathrm{Se}_{8}$

\subsection{Mossbauer investigation}

Mossbauer spectra for $3 \mathrm{c}$ and $4 \mathrm{c}$ superstructures are different because the ordered vacancies' position defines the iron atom states and $4 \mathrm{c}$ structure has additional atomic layer with vacancies. $3 \mathrm{c}$ spectra consist of three sextets, each corresponding to specific iron sublattice - A, B and C. A sublattice is bound to the iron atoms in the layers with no vacancies, B lattice is bound to the iron atoms in the layers with diagonal vacancies and $\mathrm{C}$ lattice indicates the iron atoms in the layers with other vacancies. In $4 \mathrm{c}$ structure, A sublattice is split into A1 and A2. Sublattice A1 is the same as in 3c and A2 represents iron atoms in the layers next to the additional vacancies' layer in $4 \mathrm{c}$ structure. $\mathrm{B}$ and $\mathrm{C}$ sublattices are the same as in $3 \mathrm{c}$.
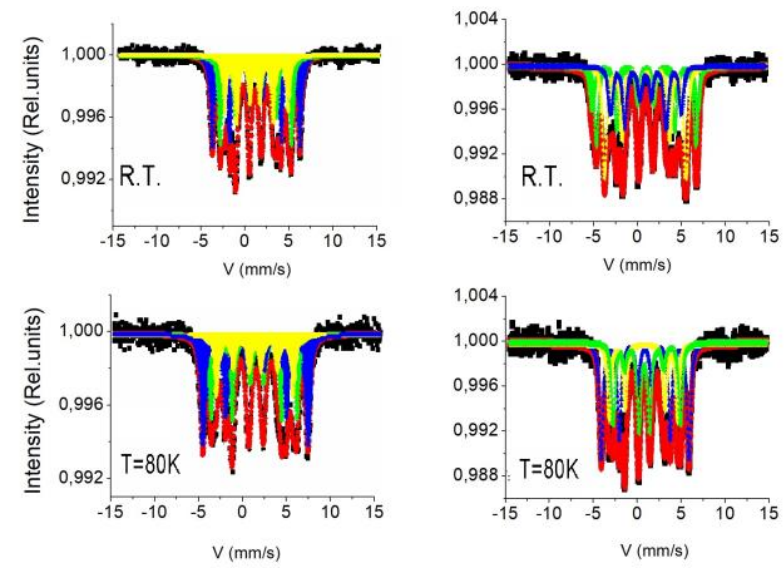

Fig.5. Mossbauer spectra obtained at room temperature and at $80 \mathrm{~K}$ for $3 \mathrm{c}-\mathrm{Fe}_{7} \mathrm{Se}_{8}$ (right) and $4 \mathrm{c}-\mathrm{Fe}_{7} \mathrm{Se}_{8}$ (left)

All the spectra for both compounds were obtained in a wide range of temperatures from $80 \mathrm{~K}$ to the room temperature (Fig.5). They were mathematically analysed and the temperature dependence of all sublattices' 
effective magnetic field for both compounds were calculated. They are shown on Fig. 6 and Fig.7.

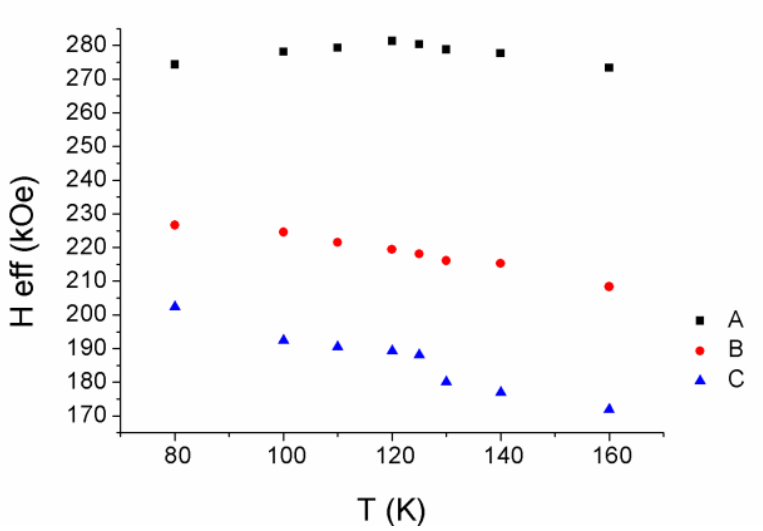

Fig.6. Sublattices effective magnetic field temperature dependence for $3 \mathrm{c}-\mathrm{Fe}_{7} \mathrm{Se}_{8}$

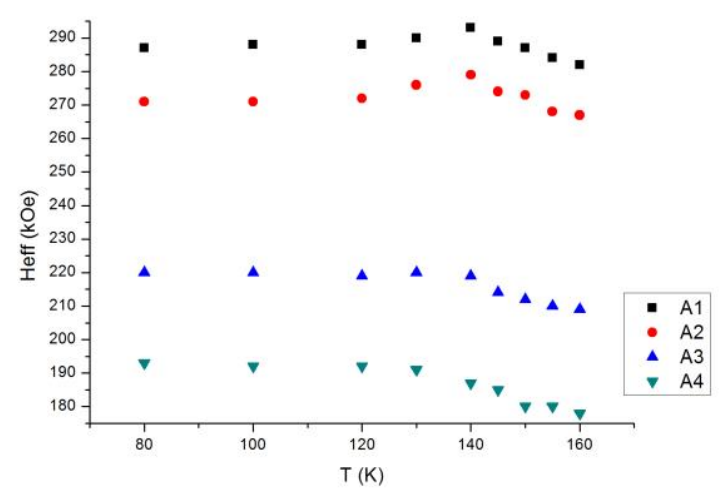

Fig.7. Sublattices effective magnetic field temperature dependence for $4 \mathrm{c}-\mathrm{Fe}_{7} \mathrm{Se}_{8}$

Such behaviour of A sublattices for both compounds is abnormal. There are peaks in temperature dependence of A sites, which indicates the beginning of magnetic disordering with the temperature lowering, that leads to a magnetic phase transition. It occurs at $120 \mathrm{~K}$ for $3 \mathrm{c}$ $\mathrm{Fe}_{7} \mathrm{Se}_{8}$ and at $135 \mathrm{~K}$ for $4 \mathrm{c}-\mathrm{Fe}_{7} \mathrm{Se}_{8}$. What is more, effective magnetic field for $4 \mathrm{c}$ A sites increased by 10 kOe comparative to $3 \mathrm{c}$, which also shows the A site spin influence increase because of vacancies adding.

\section{Conclusion}

In this work we performed the complex comparative Mossbauer and magnetic investigation of $3 \mathrm{c}-$ an $4 \mathrm{c}-$ $\mathrm{Fe}_{7} \mathrm{Se}_{8}$. We found out that adding a cell unit with the ordered vacancies makes influence on the existing ordered structure and leads to the strong increment in the magnetic moment of the whole structure. It also increases the temperature of magnetic phase transition by $15 \mathrm{~K}$. It occurs because adding the additional magnetic unit cell with four vacancies splits the A sublattice into two similar sublattices $\mathrm{A} 1$ and $\mathrm{A} 2$. It makes magnetic subsystem more flexible and increases the whole system entropy, which provides more ways for the whole $4 \mathrm{c}$ selenide to minimize it's energy without changing a ferrimagnetic order in the structure.

\section{References}

1. 14. Pan, X.; Yang, M. Q.; Fu, X et al Nanoscale 2013, 5,(9), 3601-3614

2. 15. Battaglia, C; Yin, X.; Zheng, M. et al, Nano Lett 2014,14,(2) 967-71

3. G. Li, B. Zhang, T. Baluyan et al Inorg. Chem., 2016, 55 (24), pp 12912-12922

4. A. F. Andresen and J. Leciejewicz, Journal de Physique, 1964, 25, 574-578.

5. M. Kawaminami and A. Okazaki, J. Phys. Soc. Jpn., 1970, 29, 649-655.

6. C. Boumford, and A. H. Morrish, Phys. Stat. Sol., 1974, 22, 435-444

7. I. C. Nlebedim, M. Vinitha, P. J. Praveen, D. Das and D. C. Jiles, Journal of Applied Physics, 2013, 113, 193904.

8. U. Kobler, A. Hoser, J. Englich, A. Snezhko, M. Kawakami, M. Beyss and K. Fischer, J Phys Soc Jpn, 2001, 70, 3089-3097.

9. Y. J. Tang, B. Roos, T. Mewes, S. O. Demokritov, B. Hillebrands and Y. J. Wang, Applied Physics Letters, 1999, 75, 707. 